\title{
Erosive Lichen Planus - Diagnosis and Management
}

\author{
Laliytha Kumar Bijaii, ${ }^{1,}$, Philips Mathew ${ }^{2}$, Ramya Sugumar ${ }^{2}$, Ravi David Austin ${ }^{2}$ \\ ${ }^{1}$ Department of Dentistry, Sree Balaji Medical College and Hospital, Bharath University, Chennai, India \\ ${ }^{2}$ Department of Oral Medicine and Radiology,Rajah Muthiah Dental College, Annamalai University, Chidambaram, India \\ *Corresponding author: lalithadentist@gmail.com
}

Received August 30, 2014; Revised September 30, 2014; Accepted October 12, 2014

\begin{abstract}
Oral lichen planus (OLP) is a chronic inflammatory disease of unknown etiology. It seems to affect most ethnic groups and has a prevalence of $0.02-1.2 \%$ among various populations. The diagnosis of OLP can be made from the clinical features if they are sufficiently characteristic, particularly if typical skin or other lesions are present, but biopsy is recommended to confirm the diagnosis and to exclude dysplasia and malignancy. Atrophic/erosive OLP needs appropriate treatment, due to the pain and malignancy potential. Here we report a case of erosive lichen planus in a 60 -year-old male patient in the oral cavity.
\end{abstract}

Keywords: erosive lichen planus, burning sensation, malignant transformation

Cite This Article: Laliytha Kumar Bijai, Philips Mathew, Ramya Sugumar, and Ravi David Austin, "Erosive Lichen Planus - Diagnosis and Management.” International Journal of Dental Sciences and Research, vol. 2, no. 5 (2014): 112-114. doi: 10.12691/ijdsr-2-5-3.

\section{Introduction}

Oral lichen planus (OLP) is a chronic inflammatory disease of skin and/or oral muscosa that has an immunological origin. It is seen worldwide, mostly in the fifth to sixth decades of life and is twice as common in women as in men. [1] Its clinical features are variable including reticular, papular, plaque-like, atrophic and erosive lesions.

In this paper, we present a case report of erosive lichen planus in a 60- year-old male patient in the oral cavity.

\section{Case report}

A 45-year-old female patient reported to our dental OP with a complaint of burning sensation in the mouth since 1 month. Patient was apparently normal one month back. Following which he developed burning sensation in his mouth. Burning sensation aggravated on taking hot and spicy food, during smoking and relieves spontaneously. Medical history revealed cataract surgery done one year back.

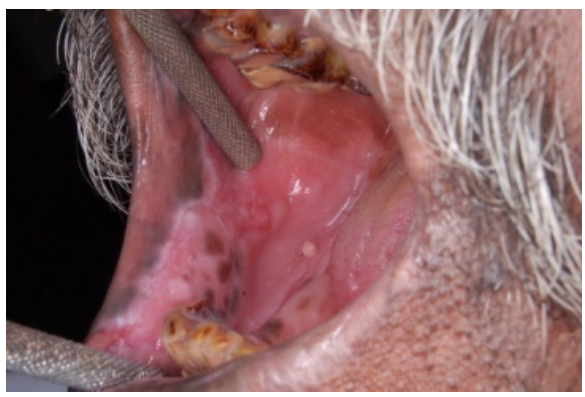

Figure 1. Erythematous area in the center with white radiating lines in the periphery evident
On intraoral examination, inspection revealed erythematous area in the center with white radiating lines in the periphery and hyperpigmented areas evident on right and left buccal mucosa, lower labial mucosa, buccal vestibule in relation to edentulous region of 46 and 47 . [Figure 1, Figure 2, Figure 3, Figure 4].

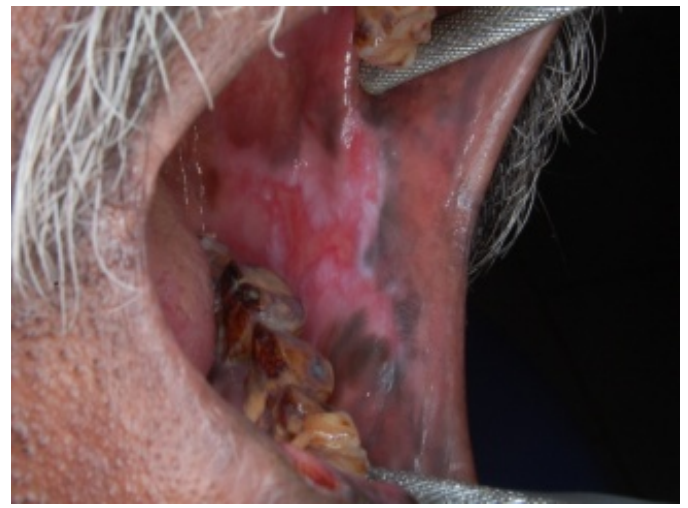

Figure 2. Erythematous area in the center with white radiating lines in the periphery evident

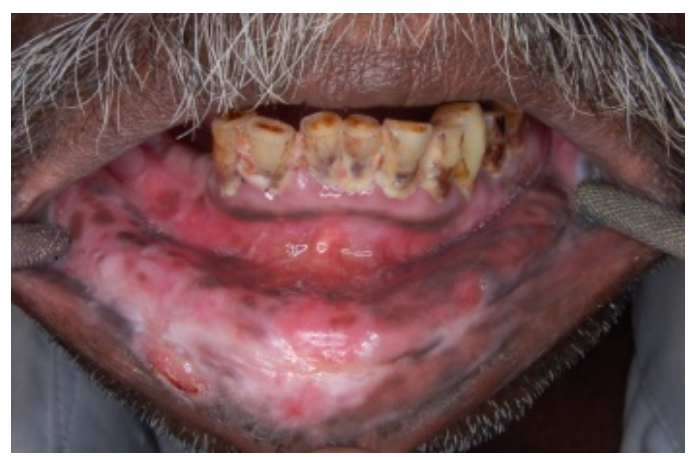

Figure 3. Erythematous area in the center with white radiating lines in the periphery evident 


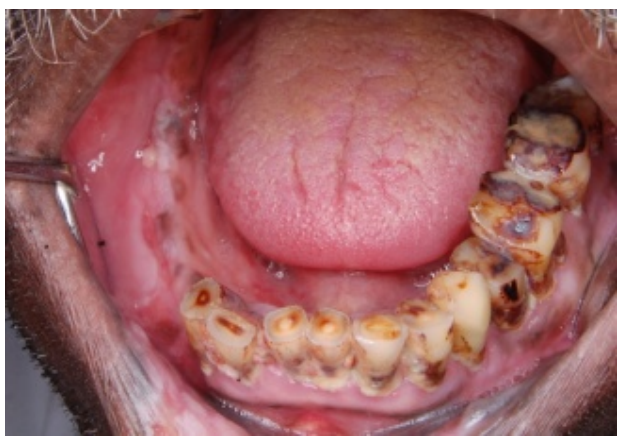

Figure 4. Erythematous area in the center with white radiating lines in the periphery evident

Based on the history and clinical examination the case was provisionally diagnosed as erosive lichen planus. The differential diagnosis considered were erythroleukoplakia, discoid lupus erythematosus, atrophic candidiasis and pemphigus vulgaris.

Histopathology of the given section revealed a nonkeratinized stratified squamous epithelium and fibrous connective tissue stroma. There is epithelial hyperplasia and in few areas ulceration is noted. The stroma of ulcerated area shows dense chronic inflammatory cell infiltration. The epithelium also shows lymphocytic infiltration. The stroma shows numerous blood capillaries. Deeper stroma shows lymphoid follicle. These findings were suggestive of lichen planus [Figure 5].

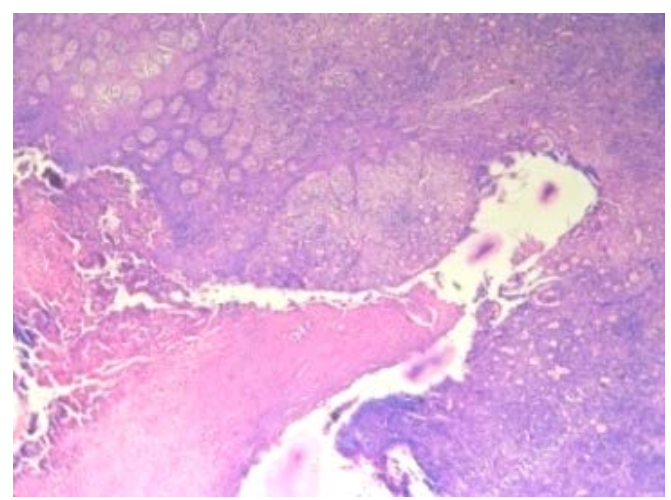

Figure 5. Histopathologic section showed non- keratinized stratified squamous epithelium and fibrous connective tissue

Patient was prescribed with Triamcinolone acetonide $0.1 \%$, Tablet prednisolone $40 \mathrm{mg}$ once daily for 7 days. Then gradual tapering of dose was done. Patient was reviewed every week. Complete resolution of symptoms was achieved after 2 weeks [Figure 7, Figure 8].

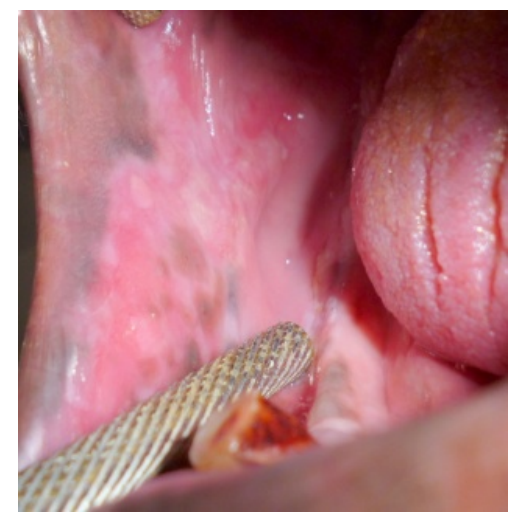

Figure 6. Post OP

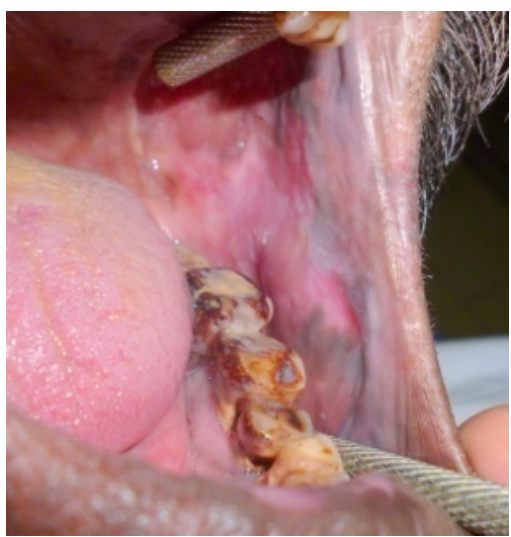

Figure 7. Post OP

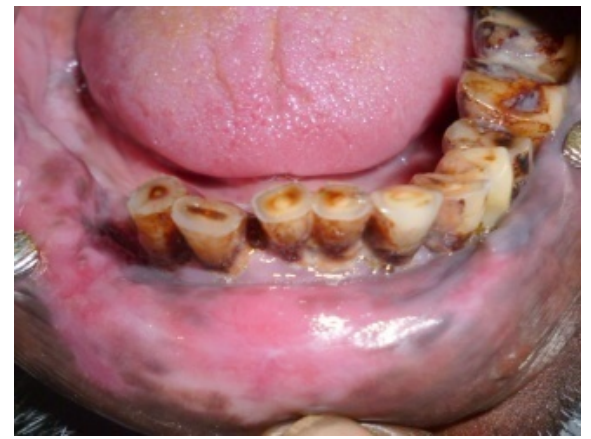

Figure 8. Post OP

\section{Discussion}

Oral lichen planus (OLP) is a relatively common chronic immunologic inflammatory mucocutaneous disorder that varies in appearance from keratotic (reticular or plaque-like) to erythematous and ulcerative.

The etiology of OLP appears to be multifactorial and complicated. The etiological factors include stress, anxiety and depression. [2,3] Familial cases of OLP have been reported and role of genetic predisposition was also considered. Watanabe $\boldsymbol{T}$ et al. stated that human leukocyte antigen (HLA) associated genetic factors play a certain role in the pathogenesis of OLP [4]. Hedberg and associates reported that epithelium involved by OLP was consistently positive for HLA-DR [5].

Clinically the oral lesions have been grouped into reticular, papular, plaque type, bullous, atrophic and erosive forms [2]. OLP usually occurs in a bilaterally symmetrical pattern, commonly involving buccal mucosa, gingiva and dorsum of the tongue.[3] The lesions are usually painless and asymtomatic, though burning sensation and pain are associated with erosive and atrophic lesions

A characteristic feature is the presence of slender white lines (Wickham's striae) radiating from the papules. In the reticular form there is a lacelike network of slightly raised gray-white lines, often interspersed with papules. The plaque type of OLP may be difficult to distinguish from leukoplakia. In some patients, however, oral lesion presents as desquamative gingivitis.

The differential diagnosis of OLP, presenting as white patches or hyperkeratoticsteriae, is broad and includes lichenoid lesions, leukoplakia, discoid lupus erythematosus, graft-versus-host disease and pemphigus vulgaris. 
Erosive OLP and lichenoid drug reactions can be indistinguishable both clinically and histopathologically. Some of the drugs commonly associated with lichenoid reactions are angiotensin-converting enzyme inhibitors, diuretics and beta-blockers [6].

The classic histopathologic features of OLP include liquefaction of the basal cell layer accompanied by apoptosis of the keratinocytes. A dense band-like lymphocytic infiltrate may be present at the interface between the epithelium and the connective tissue. Focal areas of hyperkeratinized epithelium and occasional areas of atrophic epithelium with saw toothed rete pegs [7]. Malignant potential of erosive lichen planus is more when compared to other types of lichen planus [8].

Patients with atrophic and erosive forms of OLP typically experience significant discomfort. Treatment of OLP depends on symptoms, the extent of clinical involvement, medical history and other factors. Drugs are used in two forms, topical or/and systemic. Symptomatic lesions require treatment depending on their severity which can be divided into 3 steps namely primary, secondary and tertiary line of treatment [9].

Primary line of treatment is indicated for mild to moderate symptomatic cases, topical applications of Triamcinalone acetonide $0.1 \%$ cream or Fluocinonide $0.05 \%$ gel or Dexamethazone $0.5 \mathrm{mg} / 5 \mathrm{ml}$ in orabase [9].

Secondary line of treatment is indicated for lesions which is not respond to topical treatment. In such cases Local Injection of 0.2 to $0.4 \mathrm{ml}$ Triamcinalone acetonide should be given or Systemic Prednisolone 40 to $80 \mathrm{mg}$ daily is usually sufficient to achieve a response; drug toxicity requires that it should be used only when necessary, at the lowest dose, and for the shortest time, possible [9]. Systemic Prednisolone should be taken either for brief periods of time, (5-7 days) and the dose should be reduced by $5-10 \mathrm{mg} /$ day gradually over $2-4$ weeks [9].
Tertiary line of treatment should be used for severe cases that do not respond to short term prednisolone. More protracted course of Prednisolone should be given [8]. In order to reduce the side effects of corticosteroids, Immunosuppressant such as Azathioprine 50 to $100 \mathrm{mg} /$ day, cyclosporine 50mg should be prescribed [10].

In conclusion, the current treatment modalities are inadequate in treating all patients, preventing recurrences and have significant side effects. Further clarity on the pathogenesis will aid in modifying therapeutic interventions, thus significantly can reduce the morbidity of OLP patients.

\section{References}

[1] Eisen D, Carrozzo M, Bagan Sebastian JV, Thongprasom K. Number V oral lichen planus: clinical features and management. Oral Dis 2005; 11: 338-49.

[2] Andreasen JO. Oral lichen planus. 1. A clinical evaluation of cases. Oral Surg Oral Med Oral Pathol. 1968; 25: 31-42.

[3] Scully C, El-kom M. Lichen planus: Review and update on pathogenesis. J Oral Pathol. 1985; 14: 431-58.

[4] Watanabe T, Ohishi M, Tanaka K, Sato H. Analysis of HLA antigens in Japanese with oral lichen planus. J Oral Pathol. 1986; 15: 529-33.

[5] Hedberg NM, Hunter N. Expression of HLA -DR on keratinocytes in oral lichen planus. J Oral Pathol. 1987; 16: 31-5.

[6] Bernstein ML. The diagnosis and management of chronic nonspecific mucosal lesions. J Calif Dent Assoc 1999; 27(4): 2909.

[7] Regezzi JA, Sciubba JJ. Oral pathology: Clinical pathologic correlations. 3rd ed. Philadelphia: WB Saunders; 1999.

[8] Homstrup P, Thorn JJ, Rindum J, Pindborg JJ. Malignant development of lichen planus-affected oral mucosa. J Oral Pathol 1988; 17: 219-25.

[9] Scully C, Porter SR. The clinical spectrum of desquamative gingivitis. Semin Cutan Med Surg1997; 16: 308-13.

[10] Lear JT, English JS. Erosive and generalized lichen planus responsive to azathioprine. Clin Exp Dermatol 1996; 21: 56-7. 